\begin{tabular}{|c|l|}
\hline Title & A Partial Width Calculation of OZI-A llowed Charmonium Decays in a Coupled Channel Framework \\
\hline Author(s) & Sakai, M.; Matsuda, Y.; Hirano, M.; Kato, K. \\
\hline Citation & $\begin{array}{l}\text { Few-Body Systems, 46/3), 189-198 } \\
\text { https:/doi.org/10.1007/300601-009-0060-5 }\end{array}$ \\
\hline Issue Date & 2009-09 \\
\hline Doc URL & http://hdl.handle.net/2115/43812 \\
\hline Rights & The original publication is available at www.springerlink.com \\
\hline Type & article (author version) \\
\hline File Information & FBS46-3_189-198.pdf \\
\hline
\end{tabular}

Instructions for use 


\title{
A Partial Width Calculation of OZI-Allowed Charmonium Decays in a Coupled Channel Framework
}

\author{
M. Sakai ${ }^{1, *}$, Y. Matsuda ${ }^{2, * *}$, M. Hirano ${ }^{3, * * *}$, K. Kato ${ }^{4, \dagger}$ \\ ${ }^{1}$ Department of Physics, Hokkaido University of Education at Asahikawa, 070-8621 Asahikawa, Japan \\ ${ }^{2}$ Faculty of Dairy Science, Rakuno Gakuen University, 069-8501 Ebetsu, Japan \\ ${ }^{3}$ Department of Physics, Hokkaido University of Education at Sapporo, 002-8502 Sapporo, Japan \\ ${ }^{4}$ Division of Physics, Graduate School of Science, Hokkaido University, 060-0810 Sapporo, Japan
}

\begin{abstract}
OZI-allowed partial decay widths, masses, and total decay width of charmonium states are studied in a nonrelativistic coupled-channel framework based on microscopic effective quark interactions. With the help of the complex scale transformation, the coupled channel equation is easily solved under the proper boundary condition for resonances. The obtained result as a whole is very successful and encouraging for the traditional charmonium states including $\psi(4040)$ whose features of mass and partial decay widths have been argued historically. The coupling mechanisms of these states are investigated by reducing artificially the channel coupling strengths little by little and finally turning the coupling off. The situations turn out to be quite different from what we would have naively supposed. Other solutions than the traditional charmonium states were obatined at the same time. Some of them are discussed in relation with new particles observed recently.
\end{abstract}

\section{Introduction}

In this article we study masses and Okubo-Zweig-Iizuka ${ }^{1}$ (OZI)-allowed decay widths of charmonium states. The charmonium states and their observed open decay channels that we consider are shown in Fig. 1 . In the analysis ${ }^{2}$ of OZI-allowed decays, ${ }^{1}$ importance of the nodal structure of charmonium wavefunctions was noted ${ }^{3,4}$ first by the Orsay group. The harmonic oscillator model was used for the mass spectrum and the ${ }^{3} P_{0}$ model $^{5}$ for the decay vertex. In those analyses, mass and decay dynamics were treated separately. Soon after, the Cornell group proposed a unified treatment of mass and decay based on an effective quark interaction showing the QCD features. ${ }^{6}$ Their

\footnotetext{
${ }^{*} E$-mail: m-sakai@asa.hokkyodai.ac.jp

**E-mail: y-mazda@wish.ocn.ne.jp

***E-mail: hirano@sap.hokkyodai.ac.jp

${ }^{\dagger} E$-mail: kato@nucl.sci.hokudai.ac.jp
} 


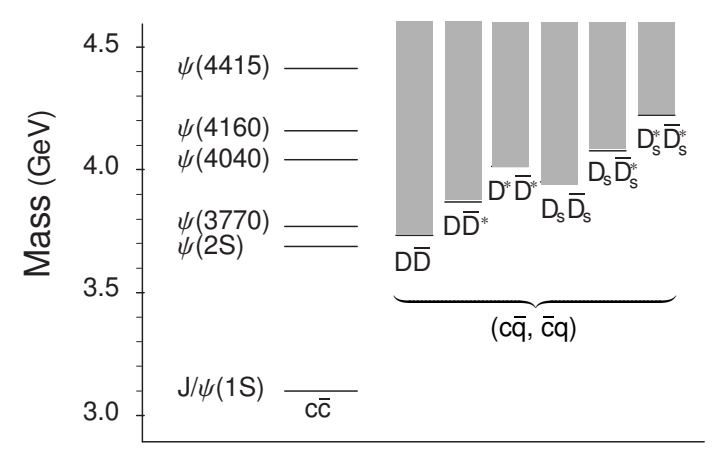

Figure 1. Spectra of the $c \bar{c}$ system and of the lower-lying charmed-meson decay channels.

numerical results, however, turned out to be not so satisfactory. Recently, the Cornell group reported that their model happened to produce good numerical results ${ }^{7}$ by using a different value of string tension from that chosen previously.

We have slightly modified $^{8}$ the Cornell model by introducing the one-bosonexchange(OBE) interaction between heavy mesons in open channels. We set up a coupled channel equation with the linear plus Coulomb potential in the confined $c \bar{c}$ channels, OBE potentials in the meson-antimeson $(M \bar{M})$ open decay channels and transition potentials in the off diagonal parts of the potential connecting the two channels. A solution of the $c \bar{c}$ channels without the decay coupling is a bound state without any decay width. A solution of the latter system without coupling is that of either a continuum state or an unbound S-matrix pole state with a large decay width. By the coupling of these channels, bound states, resonant- and pole-states other than continuum states are brought about. That is, a heavy quark-antiquark pair-state below open decay channels stays as a bound state, the one above open channel thresholds obtains a decay width and becomes a resonant state, and some of pole states with wide widths and continuum states can be pole states with comparatively narrow widths. When the total width of some of these pole states is narrow, the state can be an observable resonant state.

The coupled channel equation was transformed by the complex coordinate scale transformation ${ }^{9}$ and then we solved the transformed equation numerically. Using the framework, mass spectra and total decay widths of $\psi$ 's ${ }^{8}$ and $\Upsilon$ ''s ${ }^{10}$ have previously been studied. The partial decay width analysis needs more detailed knowledge of the wave functions. In the present analysis, not only mass and total decay width but partial decay widths are studied. By this analysis, reliability of the framework will be tested in detail.

\section{Partial Decay Width in the Coupled Channel Framework}

\subsection{Brief Survey of the Framework}

In this section we briefly outline our coupled-channel framework. For details, refer to ref. 8 of Yabusaki et al. In this paper, we only deal with the $J^{P C}=1^{--}$charmonium states. Our coupled-channel equation consists of the confined $c \bar{c}$ channels and the 6 open $M \bar{M}$ channels. The formers are two confined $c \bar{c}$ channels, one with the relative orbital angular momentum $L=0$ and the other with $L=2$. The latter open channel states we consider are those shown in Tab. 1 . Among them, the $D^{*} \bar{D}^{*}$ - and $D_{s}^{*} \bar{D}_{s}^{*}$-channels have 
two relative orbital angular momenta $l=1,3$ and the others $l=1$. These heavy mesons in the open channels are treated as elementary ones.

The radial part of our coupled channel equation is written as in the following form;

$$
\mathcal{H} \Psi(r)=\{\mathcal{T}(r)+\mathcal{V}(r)\} \Psi(r)=E \Psi(r)
$$

Here $\mathcal{T}(r)$ and $\mathcal{V}(r)$ are the kinetic and potential energy operators, respectively;

$$
\mathcal{T}(r)=\left(\begin{array}{cc}
-\frac{1}{2 \mu_{0}} \frac{\mathrm{d}^{2}}{\mathrm{~d} r^{2}} & 0 \\
0 & -\frac{1}{2 \mu} \frac{\mathrm{d}^{2}}{\mathrm{~d} r^{2}}
\end{array}\right) \text { and } \mathcal{V}(r)=\left(\begin{array}{cc}
G(r)+\frac{L(L+1)}{2 \mu_{0} r^{2}} & f(r) \\
f^{\mathrm{T}}(r) & Y(r)+\frac{l(l+1)}{2 \mu r^{2}}
\end{array}\right),
$$

where $\mu_{0}$ is the reduced mass of the $(c, \bar{c})$ system. The matrix $\mu^{-1}$ represents a diagonal matrix whose diagonal element is reciprocal of the reduced mass $\mu_{j}$ of the corresponding $j$-th open channel listed in Tab. 1.

Table 1. Open $M \bar{M}$ channels taken into account in the present analysis

\begin{tabular}{cccc}
\hline$j$-th & Open channel & $j$-th & Open channel \\
\hline 1 & $D \bar{D}$ & 4 & $D_{s} \bar{D}_{s}$ \\
2 & $\left(D \bar{D}^{*}+D^{*} \bar{D}\right) / \sqrt{2}$ & 5 & $\left(D_{s} \bar{D}_{s}^{*}+D_{s}^{*} \bar{D}_{s}\right) / \sqrt{2}$ \\
3 & $D^{*} \bar{D}^{*}$ & 6 & $D_{s}^{*} \bar{D}_{s}^{*}$ \\
\hline
\end{tabular}

The potential $G(r)$ in the $c \bar{c}$ channel is of the standard linear plus Coulombic type,

$$
G(r)=\lambda r+b-\frac{\alpha}{r},
$$

where $\lambda, b$ and $\alpha$ are the string tension, a constant potential and one-gluon exchange constant of QCD, respectively. Extending this potential, an effective quark interaction has been considered; ${ }^{6,11}$

$$
H_{\mathrm{int}}=\frac{3}{8} \int \mathrm{d}^{3} \boldsymbol{x} \mathrm{d}^{3} \boldsymbol{x}^{\prime} \bar{\psi}_{f^{\prime}}\left(\boldsymbol{x}^{\prime}\right) \Gamma_{i} \frac{\lambda^{a}}{2} \psi_{f^{\prime}}\left(\boldsymbol{x}^{\prime}\right) V_{i}\left(\left|\boldsymbol{x}^{\prime}-\boldsymbol{x}\right|\right) \bar{\psi}_{f}(\boldsymbol{x}) \Gamma_{i} \frac{\lambda^{a}}{2} \psi_{f}(\boldsymbol{x}) .
$$

The $\psi_{f}(\boldsymbol{x})$ stands for the quark field operator with the flavor $f$. It is a vector in color degree of freedom and $\lambda^{a}$ s are the color SU(3) generators. $\Gamma_{i}$ is either the $\gamma$ matrix or the unit matrix depending on the Lorentz property (vector or scalar) of $V_{i}(r)$. As $V_{i}(r)$ $(i=1,2)$, we take the Coulombic potential and the linear plus constant potential, both transforming as Lorentz vectors in this article;

$$
V_{1}(r)=-\frac{\alpha}{r}, \quad V_{2}(r)=\lambda r+b,
$$

where $G(r)=V_{1}(r)+V_{2}(r)$. By this effective interaction Hamiltonian, mass and decay of heavy quarkonium states, ${ }^{6}$ the quark condensation ${ }^{11}$ and the chiral symmetry breaking ${ }^{12}$ have been studied. The off-diagonal $f(r)$ is the transition potential connecting the $c \bar{c}$ channels and the $M \bar{M}$ channels. The explicit form $f(r)$ based on Eq. (2.4) is given in ref. 8. The diagonal $Y(r)$ is the $M \bar{M}$ potential. 
A charmonium state is expressed by a multi-component wave function $\Psi(r)$ as a sum of closed quark-antiquark components $b(r)$ and open $M \bar{M}$ components $u_{j}(r)$ :

$$
\Psi(r)=\sum_{L} b^{L}(r)+\sum_{j, l} u_{j}^{l}(r) .
$$

In this article, we consider mainly $1^{--}$charmonium states of $\psi(1 S)-\psi(4 S), \psi(1 D)$ and $\psi(2 D)$.

Now we briefly explain the formulae for evaluating partial decay widths that have been discussed by Moiseyev and Peskin. ${ }^{13}$ Their formulae are based on the complex (coordinate) scale transformation ${ }^{9}$ of the relative coordinate of the two-particle system to the complex plane;

$$
U(\theta): r \rightarrow r \mathrm{e}^{\mathrm{i} \theta} .
$$

Then the coupled-channel equation is expressed as

$$
\mathcal{H}(\theta) \Psi_{\theta}(r)=E_{\theta} \Psi_{\theta}(r),
$$

where $\mathcal{H}(\theta)=U(\theta) H U(\theta)^{-1}$, and $\Psi_{\theta}(r)=U(r) \Psi(r)$. Since $\mathcal{H}(\theta)$ is not hermitian, eigenvalues $E_{\theta}$ are not real. According to the so-called ABC-theorem, ${ }^{9}$ the bound and resonant states have $\theta$-independent real(negative) and complex eigenvalues, respectively. The real and imaginary parts of the complex resonant eigenvalue $E_{\theta}^{\mathrm{r}}=M-\mathrm{i} \Gamma / 2$ stand for mass and a half of total decay width of the system. The eigenvalue $E_{\theta}^{\mathrm{r}}$ is independent of $\theta$ if $\theta \geq \tan ^{-1}(\Gamma / 2 M)$. The continuum solutions have continuous complex energy $E_{\theta}^{\mathrm{c}}=E_{\mathrm{r}}+\mathrm{i} E_{\mathrm{i}}$ on the $2 \theta$-rotated branch cut. There is a maximum angle $\theta_{\max }$ determined by the analyticity condition of the transformed potential $V\left(r \mathrm{e}^{i \theta}\right)$; for example, $\theta_{\max }=\pi / 4$ for the Gaussian potential.

The merit of this method is that resonant-state solutions can be obtained by the same method as bound-state solutions in the $L^{2}$ integrable functions. It has been applied to systems in the field of atomic and molecular physics and then to nuclear physics. Subsequently, the method was applied to mass and total decay width evaluation of $\psi$ 's ${ }^{8}$ and $\Upsilon^{\prime}$ 's. ${ }^{10}$

\subsection{Moiseyev-Peskin Formula for Partial Decay Widths}

In the coupled channel framework, a resonance wave function $\Psi(r)$ is written as a superposition of closed quark-antiquark components $b(r)$ and open $M \bar{M}$ components $u_{j}(r)$ as in Eq. (2.6). As shown in ref. 13, the ratio of the partial widths $\Gamma_{j}$ and $\Gamma_{j^{\prime}}$ of decay of a heavy quarkonium state $\psi$ to open channels $j$ and $j^{\prime}$ in Tab. 1, respectively, is given by

$$
\Gamma_{j} / \Gamma_{j^{\prime}}=\sum_{l}\left|a_{j}^{l}\right|^{2} / \sum_{l^{\prime}}\left|a_{j^{\prime}}^{l^{\prime}}\right|^{2},
$$

where $a_{j}^{l}$ is the complex-scaled flux amplitude of the $j$-th open channel component at an asymptotic region of $r$ where only the outgoing component exists.

$$
\begin{aligned}
u_{j}^{l}\left(r \mathrm{e}^{\mathrm{i} \theta}\right) & \stackrel{r \rightarrow \infty}{\longrightarrow} a_{j}^{l} \phi\left(r \mathrm{e}^{\mathrm{i} \theta}\right), \\
\phi\left(r \mathrm{e}^{\mathrm{i} \theta}\right) & =\sqrt{\frac{\mu_{j}}{\hbar k_{j}}} \mathrm{e}^{\mathrm{i} k_{j}\left(r \mathrm{e}^{\mathrm{i} \theta}\right)},
\end{aligned}
$$


where $\phi\left(r \mathrm{e}^{\mathrm{i} \theta}\right)$ is normalized to a unit current density. The expression (2.9) is rewritten in terms of the wavefunctions $u_{j}(r)$ as

$$
\frac{\Gamma_{j}^{l}}{\Gamma_{j^{\prime}}^{l^{\prime}}}=\lim _{r \rightarrow \infty}\left|\frac{\left(k_{j}\right)^{1 / 2} \mu_{j^{\prime}} u_{j}^{l}\left(r \mathrm{e}^{\mathrm{i} \theta}\right)}{\left(k_{j^{\prime}}\right)^{1 / 2} \mu_{j} u_{j^{\prime}}^{l^{\prime}}\left(r \mathrm{e}^{\mathrm{i} \theta}\right)} \mathrm{e}^{\mathrm{i}\left(k_{j^{\prime}}-k_{j}\right) r \mathrm{e}^{\mathrm{i} \theta}}\right|^{2} \equiv \lim _{r \rightarrow \infty} R_{j^{\prime} l^{\prime}}^{j l}(r) .
$$

In our model calculation, we identify Eq. (2.12) with the value $R_{j^{\prime} l^{\prime}}^{j l}(r)$ at some $r$ in the asymptotic region. From all the ratios of the partial decay widths (2.9) and the total decay width, that is, twice of an imaginary part of energy eigenvalue, we can obtain model partial decay widths.

As the method of solving our complex-scaled coupled channel Schrödinger equation numerically, we use the renormalized Numerov method ${ }^{14}$ based on the complex coordinate scale transformation. To see reliability of this numerical method and the expression (2.12), we solved the Noro-Taylor model of a simple two-channel model potential. ${ }^{15}$ The radial Hamiltonian of this model is given as

$$
H^{\mathrm{N}-\mathrm{T}}=-\frac{\mathrm{d}^{2}}{\mathrm{~d} r^{2}}+\left(\begin{array}{rr}
-1.0 & -7.5 \\
-7.5 & 7.5
\end{array}\right) r^{2} \mathrm{e}^{-r}+\left(\begin{array}{cc}
0 & 0 \\
0 & 0.1
\end{array}\right)
$$

The model has been well investigated by several other methods ${ }^{13,15,16}$ to assert their respective usefulness of evaluating partial widths. We have calculated masses, total decay widths, and partial widths $\left(\Gamma_{1}\right.$ and $\left.\Gamma_{2}\right)$ of 12 lower lying states. Our result shows a very good agreement with previous calculations, especially with the one by the complex scaled Jost function method ${ }^{16}$ for all of the 12 pole-states. As an example, our result for the first resonant pole referred to in ref. 16 is shown and compared with those obtained by other methods in Tab. 2. Thus our method has turned out to be very encouraging and no less superior than the others.

Table 2. Mass, total width and partial decay widths $\left(\Gamma_{1}, \Gamma_{2}\right)$ of the first pole (with the narrowest width) of the Noro-Taylor model having two open channels. Numerical values are given in atomic unit (a. u.).

\begin{tabular}{lllll}
\hline & mass & total width & \multicolumn{1}{c}{$\Gamma_{1}$} & \multicolumn{1}{c}{$\Gamma_{2}$} \\
\hline Noro-Taylor $^{15}$ & 4.7682 & $1.420 \times 10^{-3}$ & $5.9 \times 10^{-5}$ & $1.361 \times 10^{-3}$ \\
Moiseyev 1 $^{13}$ & 4.7682 & $1.44 \times 10^{-3}$ & $5.1 \times 10^{-5}$ & $1.368 \times 10^{-3}$ \\
Moiseyev 2 $^{13}$ & 4.7682 & $1.44 \times 10^{-3}$ & $5.1 \times 10^{-5}$ & $1.370 \times 10^{-3}$ \\
Masui et al. $^{16}$ & 4.768197 & $1.420192 \times 10^{-3}$ & $5.111562 \times 10^{-5}$ & $1.369076 \times 10^{-3}$ \\
Our prediction & 4.768197 & $1.420200 \times 10^{-3}$ & $5.111592 \times 10^{-5}$ & $1.369084 \times 10^{-3}$ \\
\hline
\end{tabular}

\section{Evaluation of Mass, Decay Width and State Fraction}

\subsection{Re-adjustment of Model Parameters}

The string tension $\lambda$ is a parameter entering not only in the confining potential but also in the transition potentials connecting the $c \bar{c}$ confining channels and open $M \bar{M}$ channels. The original Cornell value, $\lambda=0.183 \mathrm{GeV}^{2}$ has been obtained by fitting lower 
charmonium state masses in the single channel equation. The open channel effect is very large in the Lorentz vector coupling model, and $\lambda=0.183 \mathrm{GeV}^{2}$ brings a poor agreement with the experimental data for the mass difference of $2 S$ and $1 S$ states. It was found out to be about $50 \mathrm{MeV}$ smaller than the data in our Lorentz vector coupling model. In the case of the Lorentz scalar coupling model, however, the open channel effect is smaller for these states and our previous prediction using $\lambda=0.183 \mathrm{GeV}^{2}$ turned out to be good. ${ }^{8}$ By this reason, Eichten et al. have revised the strength of the string tension to be $0.258 \mathrm{GeV}^{2}$ in their Lorentz vector coupling model and have pointed out that this revision gives rise to favorable results for the controversial $3 S$ state. $^{7}$

The present model has another parameter, the strength $Y$ of the final-state-interaction (FSI), besides the string tension $\lambda$. We have changed the string tension $\lambda$ and the strength $Y$ and $c$-quark mass in this work. These parameters are readjusted so as to reproduce the overall experimental results of the $\psi$ particles, keeping $\alpha_{c \bar{c}}=0.52, m_{u}=m_{d}=0.33$ $\mathrm{GeV}$, and $m_{s}=0.45 \mathrm{GeV}$, as was set in our previous analyses. ${ }^{8}$ These latter parameters are less important since the confining force dominates over the one-gluon-exchange force, and $m_{u}=m_{d}$ and $m_{s}$ enter only in obtaining transition potentials through the wave functions of decaying charmed and anti-charmed mesons. Among the experimental data under consideration, we noted the partial decay widths of $\psi(3 S)$ above all. Figure 2 shows the ratios of the $\psi(3 S)$ partial decay widths against $Y$. This $Y$-dependence is very sharp and it helps us to determine the strength. Parameters $\lambda, m_{c}$ and $Y$ are fixed to be $\lambda=0.20 \mathrm{GeV}^{2}, m_{c}=1.72 \mathrm{GeV}, Y=0.30$ so as to reproduce overall data of charmonium states.

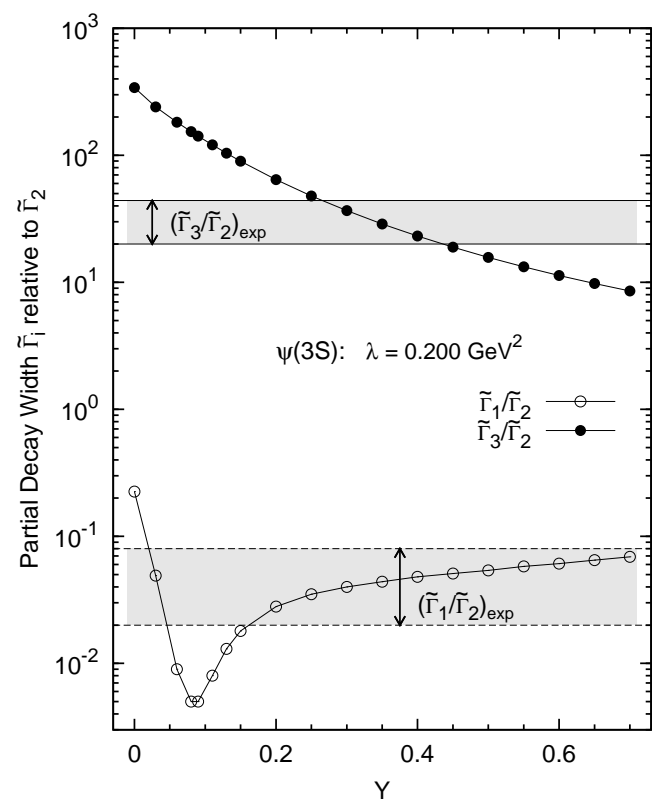

Figure 2. $Y$-dependence of the partial decay width relative to decay rate to $\left(D \bar{D}^{*}+D^{*} \bar{D}\right)$-channel. This ratio is estimated in terms of the momentum phase-volume corrected $\tilde{\Gamma}_{i}$ which differs by $p^{3}$ from $\Gamma_{i}$. Here, the index $i=1,2$ and 3 stands for $D \bar{D}, D \bar{D}^{*}+D^{*} \bar{D}$ and $D^{*} \bar{D}^{*}$ channels, respectively.

The coordinate is scaled in the complex coordinate method, and we have solved our scaled coupled channel equation. We plot the values $R_{j^{\prime} l^{\prime}}^{j l}(r)$ for all $j \neq j^{\prime}=D \bar{D}$ against $r \leq$ 


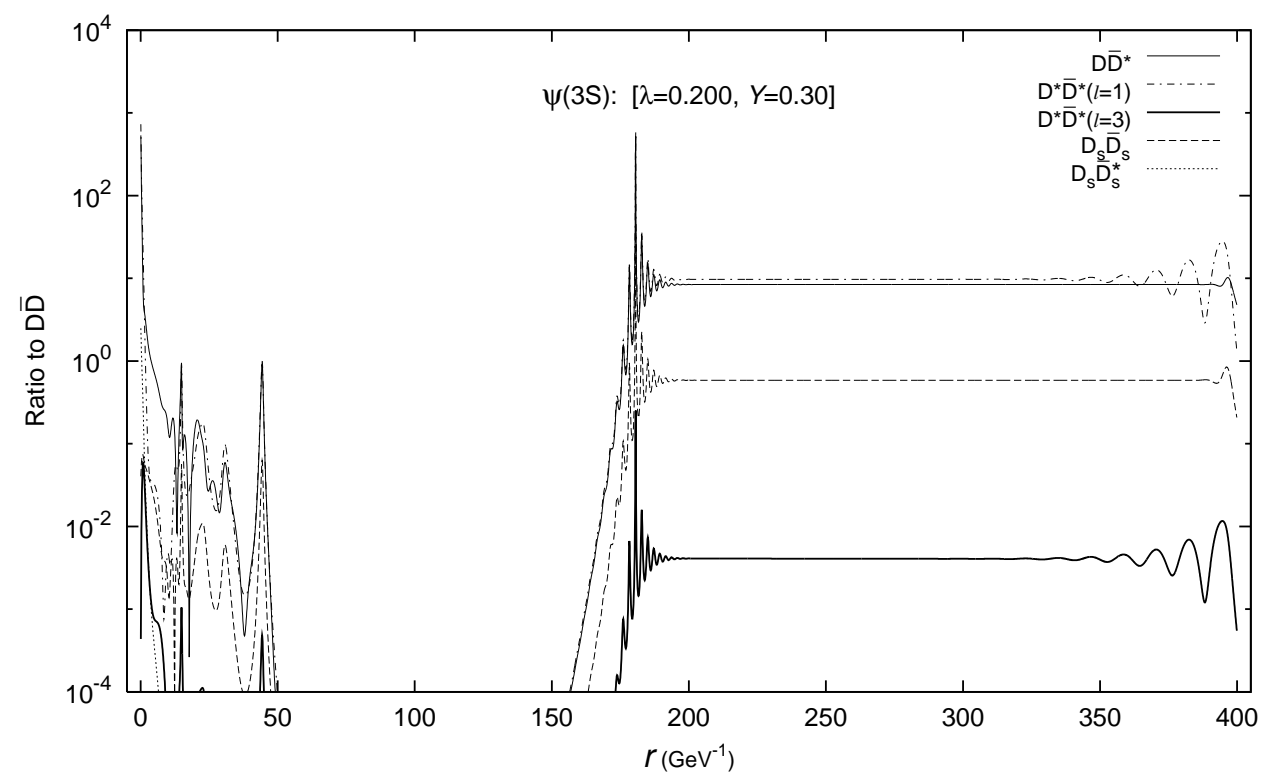

Figure 3. $R_{11}^{j l}(r)$ in Eq. (2.12), i.e., the relative strength to that of $D \bar{D}$-channel is plotted against $r$ in the case of $\psi(3 \mathrm{~S})$, where $\theta$ is set at $0.65 \mathrm{rad}$. Those for the $D_{s}^{*} \bar{D}_{s}^{*}$-channel are not seen because of its negligibly small contribution.

$400 \mathrm{GeV}^{-1}$ for $\psi(3 S)$ in Fig. 3. There is a region of stability for them to have asymptotic values in Eq. (2.12) around $r \sim(200-300) \mathrm{GeV}^{-1}$. Once the total decay width of a resonant state is obtained as an imaginary part of the complex energy eigenvalue of the complex-scaled coupled channel equation, partial decay widths are obtained from the value of the total width and the ratios, Eq. (2.9) or Eq. (2.12) for all $j$ and $j^{\prime}$.

\subsection{Mass, Decay Width and State Fraction}

The prediction of mass, total decay width and ratios among partial decay widths for traditional charmonium states are shown in Tab. 3. Of each partial decay width of the resonant states, the prediction for their dominant decay modes is listed in Tab. 4 . These results for the traditional charmonium states satisfactorily reproduce their experimental data, considering the fact that our model neglects spin-dependent forces like LS and tensor forces. Above all, $\psi(4040)$ 's feature of mass and partial decay widths, which have been viewed with great attention historically, is reproduced well. It has been difficult to reproduce its mass in the single $c \bar{c}$ channel model, and the partial decay widths have been controversial. We discuss in some detail the mechanisms of bringing our model results in the subsequent section.

In our coupled channel model, a charmonium state is expressed as a superposition of $S$-, $D$-waves, $D \bar{D}, D \bar{D}^{*}, D^{*} \bar{D}^{*}(l=1$, and $l=3)$ channels and so on. A resonant state is unbound but the complex scaled wave function both for a bound state and a resonant state is normalized to unity by the $\mathrm{ABC}$ theorem. We have calculated absolute square of each component of the complex scaled wave function and call it the fraction since they are summed up to unity. The probabilities of the traditional charmonium states finding in each of these dominant channels are listed in Tab. 5. These show that the 
Table 3. Feature of the traditional charmonium states: mass $M$, total decay width $\Gamma$ and main partial-decay width $\tilde{\Gamma}_{i}$. This quantity $\tilde{\Gamma}_{i}$ is derived by removing the $k^{3}$-phase space factor from the partial decay width $\Gamma_{i}$. An index $i$ distinguishes decaying channels as follows: $i=1$ for $D \bar{D}$, 2 for $D \bar{D}^{*}$ and 3 for $D^{*} \bar{D}^{*}$. Quantities $\tilde{\Gamma}_{1}$ and $\tilde{\Gamma}_{3}$ are given in relative to $\tilde{\Gamma}_{2}$, while $M$ and $\Gamma$ are in $\mathrm{MeV}$.

\begin{tabular}{|c|c|c|c|c|c|c|c|c|}
\hline & \multicolumn{4}{|c|}{ Experimental data } & \multicolumn{4}{|c|}{ Model prediction } \\
\hline & $M$ & $\Gamma$ & $\tilde{\Gamma}_{1} / \tilde{\Gamma}_{2}$ & $\tilde{\Gamma}_{3} / \tilde{\Gamma}_{2}$ & $M$ & $\Gamma$ & $\tilde{\Gamma}_{1} / \tilde{\Gamma}_{2}$ & $\tilde{\Gamma}_{3} / \tilde{\Gamma}_{2}$ \\
\hline $\mathrm{J} / \psi(1 S)$ & 3097 & 0 & & & 3097 & 0 & & \\
\hline$\psi(2 S)$ & 3686 & 0 & & & 3660 & 0 & & \\
\hline$\psi(3770)$ & 3771 & $23 \pm 2.3$ & & & 3807 & 11 & & \\
\hline$\psi(4040)$ & 4039 & $80 \pm 10$ & $0.05 \pm 0.03$ & $32.0 \pm 12.0$ & 4033 & 66 & 0.040 & 37 \\
\hline$\psi(4160)$ & 4153 & $103 \pm 8$ & & & 4214 & 91 & 0.052 & 137 \\
\hline
\end{tabular}

Table 4. Model prediction of dominant partial decay widths.

Masses and widths are given in $\mathrm{MeV}$.

\begin{tabular}{lccrrcc}
\hline & $M$ & $\Gamma$ & $D \bar{D}$ & $D \bar{D}^{*}$ & $D^{*} \bar{D}^{*}(l=1)$ & $D^{*} \bar{D}^{*}(l=3)$ \\
\hline $1 D$ & 3807 & 11 & 11 & 0 & 0 & 0 \\
$3 S$ & 4033 & 66 & 2 & 23 & 39 & $\sim 0$ \\
$2 D$ & 4214 & 91 & $\sim 0$ & 1 & 6 & 82 \\
\hline
\end{tabular}

Table 5. Mixing fractions of the traditional charmonium states. Integrated numbers of $\left|b^{L}\left(r \mathrm{e}^{\mathrm{i} \theta}\right)\right|^{2}$ or $\left|u_{j}^{l}\left(r \mathrm{e}^{\mathrm{i} \theta}\right)\right|^{2}$ over $r$ corresponding to Eq. (2.6) representing $S-/ D-c \bar{c}$ channel and the $M \bar{M}$ channels with angular momentum $l$ are shown in percentage(\%). Masses $M$ and total widths $\Gamma$ are given in $\mathrm{MeV}$.

\begin{tabular}{|c|c|c|c|c|c|c|c|c|c|c|c|}
\hline \multirow{3}{*}{ State } & \multirow{3}{*}{$n L$} & \multirow{3}{*}{$M$} & \multirow{3}{*}{$\Gamma$} & \multicolumn{6}{|c|}{ state fractions $(\%)$} & \multirow{3}{*}{$M_{\text {exp }}$} & \multirow{3}{*}{$\Gamma_{\exp }$} \\
\hline & & & & \multicolumn{2}{|c|}{$c \bar{c}$} & \multirow[t]{2}{*}{$D \bar{D}$} & \multirow[t]{2}{*}{$D \bar{D}^{*}$} & \multicolumn{2}{|c|}{$D^{*} \bar{D}^{*}$} & & \\
\hline & & & & $S$-wave & $D$-wave & & & $(l=1)$ & $(l=3)$ & & \\
\hline $\mathrm{J} / \psi(1 S)$ & $1 S$ & 3097 & & 96 & 0 & & 1 & 2 & & 3097 & 0 \\
\hline$\psi(2 S)$ & $2 S$ & 3666 & 0 & 79 & 6 & 2 & 6 & & & 3686 & 0 \\
\hline$\psi(3770)$ & $1 D$ & 3807 & 11 & 21 & 72 & & & & & 3770 & $23 \pm 3$ \\
\hline$\psi(4040)$ & $3 S$ & 4033 & 66 & 79 & 6 & 1 & 3 & 8 & & 4040 & $80 \pm 10$ \\
\hline$\psi(4160)$ & $2 D$ & 4214 & 91 & 32 & 63 & & & & 3 & 4159 & $103 \pm 8$ \\
\hline
\end{tabular}

usual nomination of the traditional charmonium states as being $n L(n$ : principal quantum number, $L$ : orbital angular momentum) of $c \bar{c}$ is appropriate.

The model predicted $\psi(3770)$ is predominantly of $D$-waves but contains a finite amount of $S$-wave components. This makes a finite $e^{+} e^{-}$decay width, consistent with the experimental data. ${ }^{17}$ Of the $2 D$ state, the partial decay width data is not known, but our model predicts that it is dominated by the $D^{*} \bar{D}^{*}$ decay over both $D \bar{D}$ and $D \bar{D}^{*}$ as in the $3 S$ case. However, the $l$-dependence of $D^{*} \bar{D}^{*}$ is quite different between the $3 S$ and $2 D$ states; the $l=1$ component of the $D^{*} \bar{D}^{*}$ decay is dominant for the $3 S$ state, while the $l=3$ component is dominant for the $2 D$ state. Our prediction of a small $D \bar{D}$ width of the $2 D$-state contrasts with results of $\Gamma_{D \bar{D}}^{(2 D)} / \Gamma \sim 0.5$ by Alcock et al. ${ }^{18}$ and $0.4-0.5$ by 


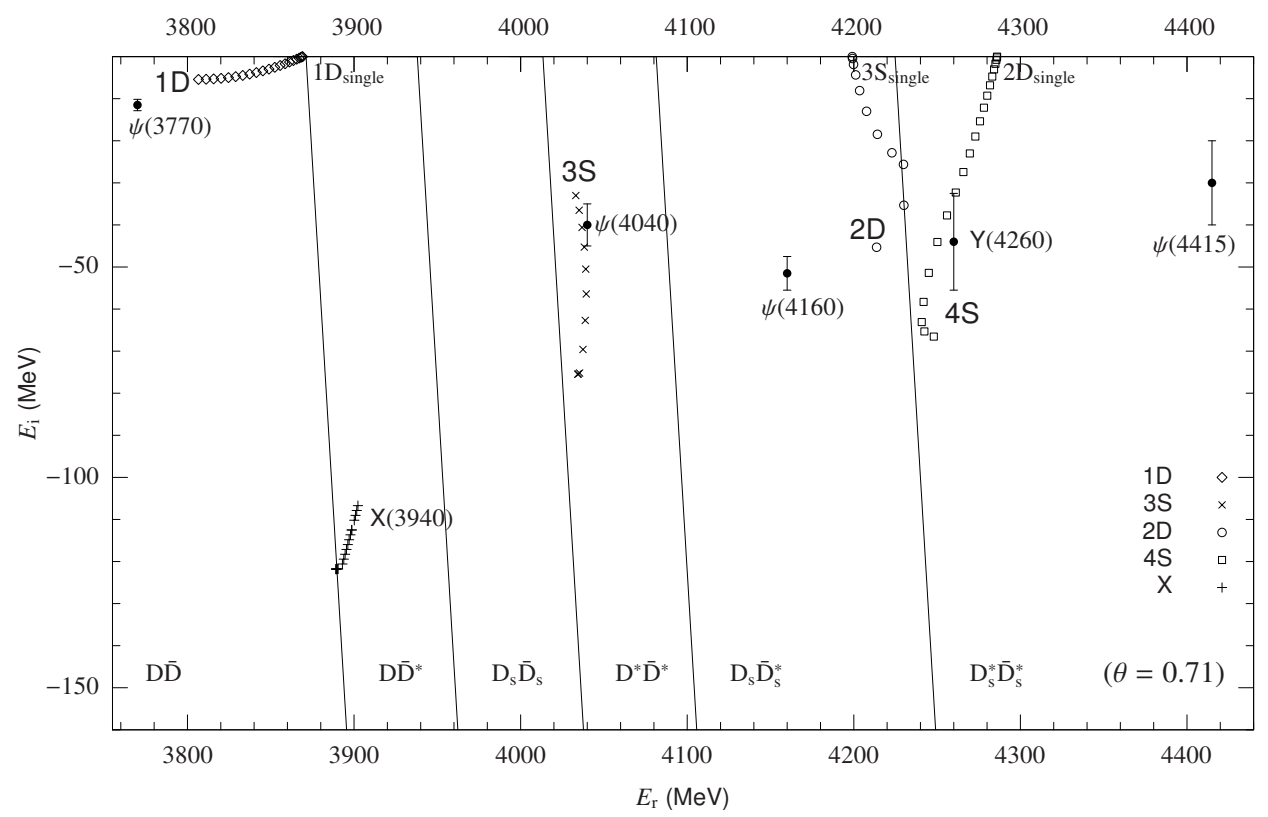

Figure 4. Dependence of energy eigenvalues, $E_{\mathrm{r}}$ and $E_{\mathrm{i}}(=-\Gamma / 2)$, on the strength parameter $f$ of the coupling when the transition potential $f(r)$ is replaced by $f f(r)$. Here, $f$ is varied through 1 to 0 .

Bradley-Robson. ${ }^{19}$ The $2 D$-state, as shown in Tab. 5, contains an $S$-wave $c \bar{c}$ component as large as $32 \%$. This is favorable since it has a much larger $e^{+} e^{-}$width $^{17}$ than one would expect for a pure $D$-wave $c \bar{c}$ state.

\section{Coupling Mechanism in the Mass Region 4040-4260 MeV}

How are numerically obtained physical charnomium states brought by the open channel couplings? The confined $c \bar{c} S$ - and $D$-channels and open $M \bar{M}$ channels are coupled by the transition potentials in our coupled channel equation. The coupled channel solutions are expressed in the linear combination of eigenstates of these single $c \bar{c}$ and open $M \bar{M}$ channels. The transition potential fixes the mixing of these single channel eigenstates. We have inquired the coupling mechanism of the obtained solutions of the coupled channel equation. For this purpose, we replace the transition potential $f(r)$ by $f f(r)$ and the coupled channel equation are solved for every strength $f$ of the replaced transition potential from the maximum $(f=1)$ given by the model down to zero (the single $c \bar{c}$ channel and $M \bar{M}$ equations).

The obtained coupled channel $1 S, 2 S$ and $1 D$ solutions are found to be reduced to the single $c \bar{c}-1 S, 2 S$ and $1 D$ channel solutions respectively when $f=0$. The higher mass states, however, are not in such corresponding order. The situation for resonant states above the lowest open channel threshold is depicted in Fig. 4. As seen from the figure, the controversial state $\psi(4040)$ behaves quite differently from other charmonium states. Namely, the other charmonium states goes to $c \bar{c}$ single channel states when $f$ tends to zero while the $\psi(4040)$ state does not. It comes very near to the $D^{*} \bar{D}^{*}$ cut for $f<0.6$ and the solution becomes $\theta$-dependent. A physically meaningful solution must 
be $\theta$ independent. The information of the state $\psi(4040)$ for $f<0.6$ is beyond power of our framework and we cannot have $f=0$ trustworthy information of the state. However, the situation of $1>f>0.6$ will suggest that the $\psi(4040)$ state is a very strong coupled state of $c \bar{c}$ channel states and $D^{*} \bar{D}^{*}$ states. The features cannot be realised at all either by a $c \bar{c}$ single channel picuture nor a $D^{*} \bar{D}^{*}$ molecular picture. ${ }^{20,21}$

Then a following question arises. What is the state that the $c \bar{c}$ single channel $3 S$ state becomes when the open channel effect is switched on? The answer by our analysis is $2 D$. The $c \bar{c}$ single channel $2 D$ state, on the other hand, becomes a dominant $c \bar{c} S$ wave state with mass about $4260 \mathrm{MeV}$. This may be identified with $Y(4260)$. As seen from Fig. 4, we may say that crossing occurs between these two states and their orbital angular momenta are changed to each other at a coupling stage through receiving the open channel effect.

Our predictions of ratios of partial decay widths for the $3 S$ and $2 D$ states are listed and compared with those obtained by previous analyses in Fig. 5. These predictions

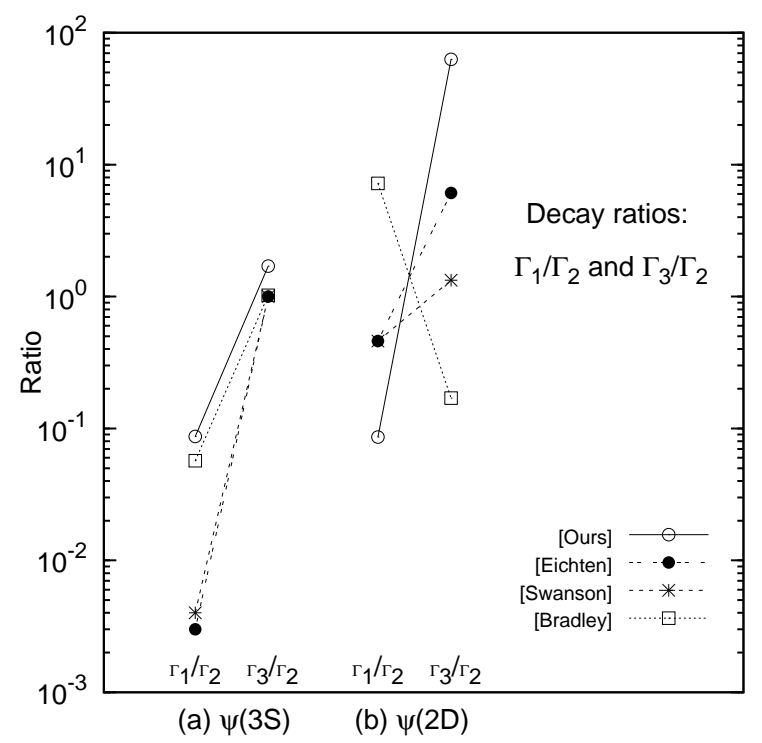

Figure 5. Ratios of partial widths among decays to $D \bar{D}\left(\Gamma_{1}\right), D \bar{D}^{*}\left(\Gamma_{2}\right)$ and $D^{*} \bar{D}^{*}\left(\Gamma_{3}\right)$ from (a) $3 S$ and (b) $2 D$. Our prediction is compared with those obtained by Eichten et al., ${ }^{7}$ Swanson et al. ${ }^{5}$ and Bradrey-Robson. ${ }^{19}$

for the $\psi(2 D)$ except that by Bradley-Robson show a tendency that $\Gamma_{3} / \Gamma_{2}$ is larger than $\Gamma_{1} / \Gamma_{2}$. It is noted that this tendency is similar to that of $\psi(3 S)$. However, the individual values of these ratios for the $\psi(2 D)$ are very dispersive among themselves. As we have not yet data on the $\psi(2 D)$ partial decays, experiments on these partial decays are highly anticipated.

\section{Discussion and Summary}

Backed by success of the present model for the traditional charmonium states, we discuss here some other states obtained by our coupled channel equation. These states and their possible assignments are shown in Tab. 6.

A solution exists near the mass region of $X(3940)^{22}$ as seen in Fig. 4. It can decay 
Table 6. Our model prediction of other charmonium states than those in Tab. 5. Masses and widths are given in MeV. The column ' $c \bar{c}$ fraction' shows $S$ - and $D$-wave components (in \%) in a bracket.

\begin{tabular}{|c|c|c|c|c|c|c|}
\hline \multirow[b]{2}{*}{ State } & \multirow[b]{2}{*}{$n L$} & \multicolumn{3}{|c|}{ Model prediction } & \multicolumn{2}{|c|}{ Experimental data } \\
\hline & & $M$ & $\Gamma$ & $c \bar{c}$ fraction & $M$ & $\Gamma$ \\
\hline$X(3940)$ & - & 3902 & 213 & {$[71$,} & 3943 & $<52$ \\
\hline$Y(4260)$ & $4 S$ & 4248 & 133 & {$[74$} & 4259 & $88 \pm 23 \pm 5$ \\
\hline$\psi(4415)$ & $5 S$ & 4561 & 30 & {$[91$,} & 4415 & $60 \pm 20$ \\
\hline
\end{tabular}

both to $D \bar{D}^{*}$ and $D \bar{D}$ states energetically, but the model predicts the $D \bar{D}^{*}$ decay dominance. The prediction of a branching fraction of more than $99 \%$ for the $D \bar{D}^{*}$ decay is consistent with the present data. Although the predicted total decay width is very large compared with the data, we identify this state as $X(3940)$ by these model features. Eichten suggests that it could be $\eta_{c}(3 S)$, but it is quite interesting that our coupled channel equation for the $1^{--}$system brings about a solution showing roughly the same features as those by experiments.

We have found a $1^{--}$solution at $M=4248 \mathrm{MeV}$ and $\Gamma=133 \mathrm{MeV}$, a little apart from the $D_{s}^{*} \bar{D}_{s}^{*}$ cut. It may be assigned to be the $\psi(4 S)^{23}$ noting that the $c \bar{c}$ component is found to be $74 \%$ in $S$-wave and $17 \%$ in $D$-wave, although the solution moves to a $c \bar{c}$ state with $L=2$ with mass about $4286 \mathrm{MeV}$ when the open channel coupling is turned off. This solution is located quite close to $Y(4260)^{24}$ whose mass and the total decay width is $4259 \mathrm{MeV}(88 \pm 23 \pm 5 \mathrm{MeV})$. Therefore, they can be identified.

We also have found a solution at $M=4560 \mathrm{MeV}$ and $\Gamma=30 \mathrm{MeV}$. This might be a candidate for the experimentally observed $\psi(4415)$. The dominant components are calculated to be $91 \% S$ and $4 \% D$. Usually, this state has been assigned as $\psi(4 S)$. By the naive potential model, the leptonic width is predicted to be larger by more than factor 2 of the observed value. Hence, $\psi(5 S)$ assignment could be a possibility. The mass turns out to be much larger than the data, but we think that this is not a serious problem. Because there will be some other open decay channels which we do not take into account, and because relativistic effects and spin dependent effects are neglected in this study.

As a whole, our coupled channel model nicely reproduces the present experimental data of $1^{--}$charmonium states. The newly observed $X(3940)$ and $Y(4260)$ are well seated in the model. We have studied the coupling mechanisms of all these states. We found that the channel coupling is so strong that the structure of fraction of an eigenstate is drastically changed by the coupling for many of the resonant states. The pictures of the mechanism revealed by the model, especially for $3 S$ and $2 D$ states are quite far from the naive picture of the charmonium model.

\section{References}

1. Okubo, S.: $\phi$-meson and unitary symmetry model. Phys. Lett. 5, 165 (1963)

Zweig, G.: An $\mathrm{SU}_{3}$ model for strong interaction symmetry and its breaking ; Part II. CERN Report No. 8419/Th (1964), 412(unpublished) 
Iizuka, J., Okada, K., Shito, O.: Systematics and phenomenology of boson mass levels. III. Prog. Theor. Phys. 35, 1061 (1966)

2. For the recent review of the OZI decay analyses of charmonium states, see Swanson, E. S.: The new heavy mesons: A status report. Phys. Rep. 429, 243 (2006)

3. Le Yaouanc, A., Oliver, L., Pène, O., Raynal, J. -C.: Strong decays of $\psi^{\prime \prime}(4.028)$ as a radial excitation of charmonium. Phys. Lett. B 71, 397 (1977)

4. Le Yaouanc, A., Oliver, L., Pène, O., Raynal, J. -C.: Why is $\psi(4.414)$ so narrow? Phys. Lett. B 72, 57 (1977)

5. For the recent analysis by the refined ${ }^{3} P_{0}$ model, see Barnes, T., Godfrey, S., Swanson, E. S.: Higher charmonia. Phys. Rev. D 72, 054026 (2005)

6. Eichten, E., Gottfried, K., Kinoshita, T., Lane, K. D., Yan, T. M.: Interplay of confinement and decay in the spectrum of charmonium. Phys. Rev. Lett. 36, 500 (1976); Charmonium: The model. Phys. Rev. D 17, 3090 (1978); Charmonium: Comparison with experiment. Phys. Rev. D 21, 203 (1980)

7. Eichten, E., Lane, K., Quigg, C.: New states above charm threshold. Phys. Rev. D 73, 014014 (2006)

8. Yabusaki, N., Katō, K., Hirano, M., Sakai, M., Matsuda, Y.: Mass shifts and decay widths of $\psi$ mesons due to OZI-allowed decay channels. Few-Body Syst. 28, 1 (2000)

9. Aguilar, J., Combes, J. M.: A class of analytic perturbations for one-body Schrödinger hamiltonians. Commun. Math. Phys. 22, 269 (1971)

Balslev, E., Combes, J. M.: Spectral Properties of many-body Schrödinger operators with dilatation-analytic interactions. Commun. Math. Phys. 22, 280 (1971)

Simon, B.: Quadratic form techniques and the Balslev-Combes theorem. Commun. Math. Phys. 27, 1 (1972)

For review of the complex scaling method, see Ho, Y. K.: The method of complex coordinate rotation and its applications to atomic collision processes. Phys. Rep. 99, 1 (1983)

10. Yabusaki, N., Hirano, M., Katō, K., Sakai, M., Matsuda, Y.: Masses and OZIallowed decay widths of $\gamma$ states in a coupled channel model. Prog. Theor. Phys. 106, 389 (2001)

11. Bicudo, Pedro J. de A., Ribiero, Jose E. F.: Current-quark model in a ${ }^{3} P_{0}$ condensed vacuum. Phys. Rev. D 42, 1611 (1990)

12. Amer, A., Le Yaouanc, A., Oliver, L., Pène, O., Raynal, J. -C.: Instability of the chiral-invariant vacuum for a confining potential. Phys. Rev. Lett. 50, 87 (1983)

Le Yaouanc, A., Ono, S., Oliver, L., Pène, O., Raynal, J. -C.: Spontaneous breaking of chiral symmetry for confining potentials. Phys. Rev. D 29, 1233 (1984)

Le Yaouanc, A., Oliver, L., Pène, O., Raynal, J. -C.: Quark model of light mesons with dynamically broken chiral symmetry. Phys. Rev. D 31, 137 (1985) 
13. Moiseyev, N., Peskin, U.: Partial widths obtained by the complex resonancescattering theory. Phys. Rev. A 42, 255 (1990)

14. Johnson, B. R.: New numerical methods applied to solving the one-dimensional eigenvalue problem. J. Chem. Phys. 67, 4086 (1977); The renormalized Numerov method applied to calculating bound states of the coupled-channel Schroedinger equation. J. Chem. Phys. 69, 4678 (1978)

15. Noro, T., Taylor, H. S.: Resonance partial widths and partial photodetachment rate using the rotated-coordinate method. J. of Phys. B13, L377 (1980)

16. Masui, H., Aoyama, S., Myo, T., Katō, K.: Partial decay widths in coupled-channel systems with the complex-scaled Jost function method. Prog. Theor. Phys. 102, 1119 (1999)

17. Particle Data Group: Review of particle physics. J. of Phys. G33, 765 (2006)

18. Alcock, J. W., Burfitt, M. J., Cottingham, W. N.: A string-breaking model of heavy meson decays. Z. Phys. C25, 161 (1984)

19. Bradley, A. Robson, D.: Charmonium above charm threshold - the simple approach. Phys. Lett. B 93, 69 (1980)

20. Voloshin, M. B. Okun, L. B.: Hydronic molecules and the charmonium atom. JETP Lett. 23, 333 (1976)

21. De Rújula, A., Georgi, H., Glashow, S. L.: Is charm found? Phys. Rev. Lett. 37, 398 (1976); Molecular charmonium: A new spectroscopy? Phys. Rev. Lett. 38, 317 (1977)

22. Abe, K., et al. (Belle Collaboration): Observation of a charmoniumlike state produced in association with a $J / \psi$ in $e^{+} e^{-}$annihilation at $\sqrt{S} \approx 10.6 \mathrm{GeV}$. Phys. Rev. Lett. 98, 082001 (2007)[arXiv: hep-ex/0507019].

23. Llanes-Estrada, E. J.: Y(4260) and possible charmonium assignment. Phys. Rev. D 72, 031503 (R) (2005)

24. Aubert, B., et al. (BABAR Collaboration): Observation of a broad structure in the $\pi^{+} \pi^{-} \mathrm{J} / \psi$ mass spectrum around $4.26 \mathrm{GeV} / c^{2}$. Phys. Rev. Lett. 95, 142001 (2005) Coan, T. E., et al. (CLEO Collaboration): Charmonium decays of $Y(4260), \psi(4160)$, and $\psi(4040)$. Phys. Rev. Lett. 96, 162003 (2006)

Abe, K., et al. (Belle Collaboration): Study of the $Y(4260)$ resonance in $e^{+} e^{-}$collisions with initial state radiation at Belle. BELLE-CONF-0610, hepex/0612006(2006) 